\title{
Competing Glauber and Kawasaki Dynamics
}

\author{
Simone Artz and Steffen Trimper \\ Fachbereich Physik \\ Martin-Luther-Universität \\ D-06099 Halle Germany
}

(March 15, 2018)

\begin{abstract}
Using a quantum formulation of the master equation we study a kinetic Ising model with competing stochastic processes: the Glauber dynamics with probability $p$ and the Kawasaki dynamics with probability $1-p$. Introducing explicitely the coupling to a heat bath and the mutual static interaction of the spins the model can be traced back exactly to a Ginzburg Landau functional when the interaction is of long range order. The dependence of the correlation length on the temperature and on the probability $p$ is calculated. In case that the spins are subject to flip processes the correlation length disappears for each finite temperature. In the exchange dominated case the system is strongly correlated for each temperature.
\end{abstract}

05.40.+j, 05.50.+q, 64.60.Ht,82.20.Mj 


\section{INTRODUCTION}

The time evolution of nonequilibrium Ising spin systems is of interest in particular when the spins are subject to different dynamics 1 . Initiated by Glauber stochastic model had been considered in which each spin can flip with a certain transition rate constructed in such a way that detailed balance is fulfilled. The system has to be in contact with a heat bath not specified in detail. The bath yields the energy for such a stochastic flip-process where every spin can flip in a first view independently on the orientation of neighbouring spins. In general it is assumed that the dynamics of the whole system is governed by a stochastic master equation. Recently, a very powerful method in analysing master equations was proposed 14 . It based on a quantum formulation of the underlying master equation written in terms of creation and annhiliation operators. Originally, the method had been formulated for Bose operators 79. More appropriate, in particular for problems with an exclusive dynamics, is the application of quantum Pauli operators which allows exact solutions for a whole class of one dimensional systems, for a review seet5. We have proposed an alternative way to include the coupling to a heat bath $\mathbf{E}$ explicitely. The method has been applied to study a spin-facilitated model 17 where the dynamics is restricted by specific constraints. It can be also used to find the above mentioned transition probability 18 which is not unique. It should be emphasized that the method is determined by the algebraic properties of the operators where an extension to a p-state model 19 and to a Q-statistics is straightforwarded.

Using such a Fock space formulation of the master equation we analyse a model where the system experiments with propability $p$ single spin flips (Glauber dynamics 1 ) and with propability $1-p$ spin exchange processes (Kawasaki dynamics6). The coupling to a heat bath with fixed temperature $T$ is included and the static interaction between the spins is assumed to be of infinite range21. Whereas the Glauber kinetics is always related to a change of the order parameter (nonconserved order parameter), the Kawasaki dynamics simulates the flux of energy into the system (conserved order parameter). In a continuous approach the model consists of a combination of model A and model B within the classification proposed by Hohenberg and Halperin 22.

There is a great effort in analysing such nonequilibrium spin systems with competing dynamics. Gonzalez-Miranda et a ${ }^{23}$ had studied a kinetic Ising model where Glauber and Kawasaki dynamics drive the system simultaneously. Using Monte Carlo simulations in two dimensions they found the phase diagram in the plane temperature versus the rate of Kawasaki process, here $1-p$. It reveals a line of continuous transitions between the ferro-and the paramagnetic phases. Assuming that the temperature depends on $p$ there appears for low temperatures a nonequilibrium tricritical point which had been confirmed by Dickmanes employing the dynamic pair approximation. In the present paper, the rate $p$ and the temperature $T$ are assumed to be independent variables.

Another interesting feature observed is the occurence of self organization phenomena25. Increasing the energy flux the system changes continuously from the ferromagnetic to a paramagnetic state. A further increase of flux drives the system into an antiferromagnetic phase which has been confirmed numerically at infinite temperature 3 and for finite temperatures 3 . Based on a Monte Carlo simulation Grandi et all was sucessful in calculating the critical exponents when the Kawasaki dynamics dominats the behaviour of the system. An analyt- 
ical approach has recently given by Ma et all

Here, we are interested in a quantum formulation of the problem using a Fock space representation of the master equation. The model can be attributed to the standard Ginzburg Landau functional exactly when the static interaction is realized by an infinite range model. Calculating the correlation function we find the correlation length depending on the independent variables temperature $T$ and the rate $p$ which is the probabilty that the Glauber dynamics is realized. In the model there is no indication for a tricritical point.

\section{FOCK SPACE REPRESENTATION}

The dynamics is govered by the master equation written in the symbolic form

$$
\partial_{t} P(\vec{n}, t)=L^{\prime} P(\vec{n}, t)
$$

Here $P$ is the probability that a certain configuration in terms of a lattice gas representation, $\vec{n}=\left(n_{1}, n_{2} \ldots n_{N}\right)$, is realized at time $t$. The evolution operator $L^{\prime}$ specified below is given by competing flip and exchange processes. The variables $n_{i}$ are related to the occupation number operators with eigenvalues 0 and 1 . Hence, the problem is to formulate the dynamics in such a way that this constraint is taken into account 15 . The situation in mind can be analyzed in a seemingly compact form using a Fock space representation of the master

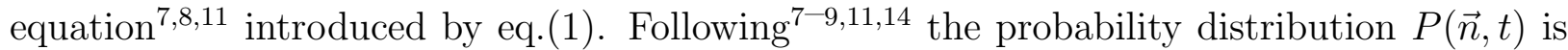
related to a state vector $|F(t)\rangle$ in a Fock-space according to $P(\vec{n}, t)=\langle\vec{n} \mid F(t)\rangle$ where the basic-vectors $|\vec{n}\rangle$ are composed of second quantized operators. The master equation (1) can be transformed into an equivalent equation in a Fock-space

$$
\partial_{t}|F(t)\rangle=L|F(t)\rangle
$$

where the operator $L^{\prime}$ in eq.(1) is mapped onto the operator $L$. Up to now the procedure is independent on the used operators. Originally, the method had been applied for the Bose case 19. Recently, an extension to restricted occupation numbers (two discrete orientations) was proposed 10 based upon a Pauli-operator representation. These operators commute at different sites and anti-commute at the same lattice site. A further extension to an $\mathrm{p}$-fold occupation number is possible 19 .

The relation between the quantum-like formalism and the probability approach is given by

$$
|F(t)\rangle=\sum_{n_{i}} P(\vec{n}, t)|\vec{n}\rangle
$$

It had been shown by Doid that the average of an arbitrary physical quantity $B(\vec{n})$ can be calculated using the average of the corresponding operator $\hat{B}(t)$ in according to

$$
\langle\hat{B}(t)\rangle=\sum_{n_{i}} P(\vec{n}, t) B(\vec{n})=\langle s|\hat{B}| F(t)\rangle
$$

with the state function $\langle s|=\sum\langle\vec{n}|$. Using the relation $\langle s| \hat{L}=0$ the evolution equation for an operator $\hat{B}$ can be written in the form

$$
\partial_{t}\langle\hat{B}\rangle=\langle s|[\hat{B}, \hat{L}]| F(t)\rangle
$$


It should be noted that all dynamical equations govering the classical problem are determined by the commutation rules of the underlying operators and the structure of the evolution operator $L$. In our case the dynamics of the model is given by spin-flip processes indicating a change of the local spin orientation and through the Kawasaki exchange dynamics. The evolution operator for a local single flip-process read\$16

$$
L_{f}=\sum_{i}\left[\lambda\left(1-d_{i}\right) d_{i}^{\dagger}+\gamma\left(1-d_{i}^{\dagger}\right) d_{i}\right]
$$

where $\lambda$ and $\gamma$ are state independent flip rates. A generalization to flip processes with restriction is discussed in 17

The operators $d_{i}$ and $d_{i}^{\dagger}$ fulfil the commutation rule of Pauli-operators. The occupation number operator $n_{i}=d_{i}^{\dagger} d_{i}$ is related to the Ising spin variable by $S_{i}=\frac{1}{2}-n_{i}$.

The evolution operator for spin conserving exchange processes reads

$$
L_{e}=\frac{\tilde{\mu}}{2} \sum_{<i, j>}\left[\left(1-d_{i} d_{j}^{\dagger}\right) d_{i}^{\dagger} d_{j}+\left(1-d_{j} d_{i}^{\dagger}\right) d_{j}^{\dagger} d_{i}\right]
$$

with the exchange rate $\tilde{\mu}$.

The complete dynamics is given by a superposition

$$
L=p L_{f}+(1-p) L_{e}
$$

The quantity $p$ represents the probability that the spins follow the Glauber dynamics whereas $1-p$ characterizes the amount of spins which are subjected to an exchange process.

Up to now the flip or exchange processes may be performed independently on the enviroment in which the system is embedded and on the static interaction between the spins. The explicit coupling to a heat bath and the inclusion of the static interaction can be realized, see 16 , by replacing the evolution operators $L_{f}$, eq.(6), by

$$
\begin{aligned}
L_{f}= & =\nu \sum\left[\left(1-d_{i}\right) \exp (-\beta H / 2) d_{i}^{\dagger} \exp (\beta H / 2)\right] \\
& +\left[\left(1-d_{i}^{\dagger}\right) \exp (-\beta H / 2) d_{i} \exp (\beta H / 2)\right]
\end{aligned}
$$

Here, $\nu$ is a new hopping rate, $\beta=T^{-1}$ is the inverse temperature of the heat bath and $H$ is the Hamiltonian describing the static interaction between the spins. In general the inclusion

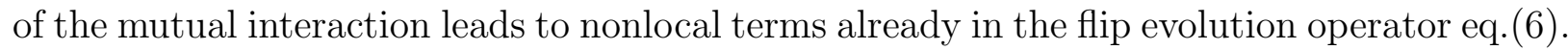
In case of the exchange dynamics the evolution operator $L_{e}$, eq.(17) should be rewritten in the form

$$
\begin{aligned}
L_{e} & =\frac{\mu}{2} \sum_{<i, j>}\left[\left(1-d_{i} d_{j}^{\dagger}\right) \exp (-\beta H / 2) d_{i}^{\dagger} d_{j} \exp (\beta H / 2)\right] \\
& +\left[\left(1-d_{j} d_{i}^{\dagger}\right) \exp (-\beta H / 2) d_{j}^{\dagger} d_{i} \exp (\beta H / 2)\right]
\end{aligned}
$$

where $\mu$ is the exchange rate. 


\section{INFINITE RANGE MODEL}

The thermalization introduced by eqs.(9, 10), is complete different to the conventional approach due to Glauber, sed6. The Hamiltonian $H$, specified below by eq.(11), mediates the coupling to the bath at the fixed temperature $T$ and to the mutual interaction present also in the static limit. Physically, the replacement of the operator $d_{i}^{\dagger}$ by $\exp (-\beta H / 2) d_{i}^{\dagger} \exp (\beta H / 2)$ in (9) means that a flip-process is realized with a weighting rate $\exp (\beta H / 2)$. After performing the flip, manifested by $d_{i}^{\dagger}$, the final state is related to the weighting rate $\exp (-\beta H / 2)$ consistent with the fact that only single flip-processes are taken into account. Hence, the procedure simulates in an analytical manner Monte Carlo steps. Due to the thermalization a spin flip is not independent on the orientation of the other spins. Instead the process is self organized by the static coupling of the spins themselves.

As the simplest case we consider an infinite range model introduced by Kac21. By reason of the long range interaction the model exhibits an exact static solution according to the mean field approximation. The Hamiltonian is defined by

$$
H=-\frac{J}{2 N} \sum_{1 \leq i<j \leq N} S_{i} S_{j}
$$

Using this Hamiltonian in the evolution operator eq.(8) there appears a conflicting situation between the local spin flip process and the infinite range interaction manifested by eq.(11). Whereas the long range static interaction favours a reordering of many spins the dynamics is refered to single spin flip processes. The situation is comparable with those known from spin glasses where the local disorder leads to restrictions within the dynamical processes.

\section{DYNAMICAL EQUATION}

Using the algebraic properties of Pauli-operators the thermalized evolution operators $L_{f}(9)$ is rewritten as:

$$
\begin{aligned}
L_{f} & =\nu \sum_{i}\left[\lambda\left(1-d_{i}\right) d_{i}^{\dagger} e^{-E}+\left(1-d_{i}^{\dagger}\right) d_{i} e^{E}\right] \\
\text { with } \quad E & =\frac{J}{2 T N} \sum_{j} S_{j}
\end{aligned}
$$

Different to the short range model (nearest neighbour coupling) the energy $E$ depends on the values of all other spins. It should be noted, that $E$ is obtained if terms with $i=j$ are not taken into consideration in eq.(11). When the diagonal terms are regarded the dynamical approach does not reproduce the correct stationary state. Within a static consideration the mentioned exclusion is irrelevant.

As the result the flip process is a thermal activated one realized with a certain rate depending on the energy $E$ including the temperature. Within the infinite range model it is easy to derive that the exchange process is not thermalized. The corresponding terms are canceled out mutually.

Taking into account the analytical form of $L_{f}$ and $L_{e}$ and using eq.(5), the evolution equation for the order parameter can be obtained: 


$$
\partial_{t}\left\langle S_{r}\right\rangle=\nu p\left(\langle\sinh E\rangle-2\left\langle S_{r} \cosh E\right\rangle\right)+\mu(1-p) \sum_{l(r)}\left\langle S_{l}-S_{r}\right\rangle
$$

where $l(r)$ means summation over all nearest neighbours of the lattice site $r$. Note that the first part, proportional to $p$, is originated from the flip process where the second one, proportional to $1-p$, can be attributed to the exchange dynamics. The situation is different to the conventional analysis where the flip processes contribute to spatial correlations, too. The evolution equation for the higher order terms appearing in eq.(13) could be calculated in a straightforward manner, seet 8 . However, in the large $N$-limit those terms has not to be evaluated provided the system is an ergodic one. It results

$$
\lim _{N \rightarrow \infty} E=\langle E\rangle=\frac{J}{2 T}\left\langle S_{r}\right\rangle
$$

Consequently, eq.(13) becomes a closed equation for the order parameter itself. In the vicinity of the phase transition the present approach leads to a Ginzburg Landau functional, see for instance22. In a continuous approximation for $\varphi(\vec{x}, t)=\left\langle S_{r}(t)\right\rangle$ it reads

$$
\begin{aligned}
\partial_{t} \varphi & =\nu p(\sinh (\epsilon \varphi)-2 \varphi \cosh (\epsilon \varphi))+\mu(1-p) \nabla^{2} \varphi \\
\text { with } \quad \epsilon & =\frac{J}{2 T} \equiv \frac{2 T_{c}}{T}
\end{aligned}
$$

The correlation function can be calculated within an expansion in terms of the order parameter $\left\langle S_{r}(t)\right\rangle$. In lowest order we find

$$
C_{0}(\omega, \vec{k}) \simeq \frac{1}{-i \omega+k^{2}+\xi^{-2}}
$$

The correlation length $\xi$ depends on the concentration $p$ and $T-T_{c}$ in the following manner

$$
\xi=\sqrt{\frac{1-p}{p}} \xi_{0} \quad \text { with } \quad \xi_{0}^{2} \simeq \frac{l^{2} \mu}{2 \nu\left(1-T_{c} / T\right)}
$$

The result can be easily interpreted. If the probability $p=1$ all spins are subject to a stochastic flip-process. But, due to the long range interaction, static fluctuations are complete suppressed. The long range force tends to align all spins parallel preventing spatial fluctuations. Consequently, the system is not able to establish spatial correlations, hence the correlation length is zero. In the opposite case $p \rightarrow 0$, when the whole spins are subject to an exchange dynamics, all neighbouring pairs of spins tend to fix their orientation (spin conservation). Then, the fluctuations are extremly strong and for $p=0$ the system remains in a strongly correlated state for finite temperatures. As the result the correlation length goes to infinity for each temperature. In the intermediated region $0<p<1$ the correlation length is a product of a $p$-dependent part and a temperature-dependent part originated from the Ginzburg Landau functional. As already emphasized, the situation is complete different to the conventional approach where the energy $E$ is a local energy leading to additonal spatial fluctuating terms originated from the spin-flip terms. One can also estimate that the inclusion of higher order fluctuations does not change the situation. A renormalization group procedure based on a Ginzburg Landau expansion starts with the correlation function $C_{0}$ obtained in (16). Such an approach yields only a modified critical exponent for the correlation length $\xi_{0}$ in eq.(17). There is also no indication for a tricritical point. 


\section{CONCLUSIONS}

In this paper we have traced back a model with competing Glauber and Kawasaki dynamics to a Ginzburg Landau functional where the correlation length depends explicitely on the amount of spins which undergo single flip processes and on the temperature. This result could be achieved by a coupling to a heat bath and by the consideration of the mutual static interaction between the spins. When this interaction is of long range order we obtain a closed set of equations for the order parameter. As the result of several conflicting situations, long range static force versus a single spin flip process or nearest neighbour exchange coupling, or Glauber versus Kawasaki dynamics the correlation length of the system depends on the probability $p$ which is a measure of the amount of spins which are subject to a Glauber process. It should be emphasized that the model is neither model A nor model B in the conventional classification22. Different to this classification the Ginzburg Landau functional consists of two parts, a nonconserving and spatial independent part and a diffusive (conserving) one originated by the exchange dynamics. 


\section{REFERENCES}

${ }^{1}$ B.C.S.Grandi and W. Figueiro, Phys.Rev. E 535484 (1996).

${ }^{2}$ B.C.S.Grandi and W. Figueiro, Phys.Rev. E 565240 (1997).

${ }^{3}$ B.C.S.Grandi and W. Figueiro, Physica A 234764 (1997).

${ }^{4}$ Y.-Q. Ma and J.-W. Liu, Phys.Lett A 238159 (1998).

${ }^{5}$ R.J. Glauber, J.Math.Phys. 4294 (19963).

${ }^{6}$ K. Kawasaki, in Phase Transitions and Critical Phenomena, C. Domb and M.S. Green, eds. Vol. 2 (Academic Press, London, 1972).

7 J. Doi M, Phys. A: Math. Gen. 9 1465, 1479 (1976).

${ }^{8}$ P. Grassberger and M. Scheunert Fortschr. Physik 28547 (1980).

${ }^{9}$ L. Peliti, J. Physique 461469 (1985).

${ }^{10}$ L.H. Gwa and H. Spohn, Phys. Rev. Lett 68725 (1992).

${ }^{11}$ S. Sandow and S. Trimper, Europhys. Lett., 21799 (1993).

12 G. Schütz and E. Domany, J. Stat. Phys. 72277 (1993).

${ }^{13}$ G. Schütz and S. Sandow, Phys. Rev. E 492726 (1994).

${ }^{14}$ F.C. Alcaraz, M. Droz, M. Henkel, and V. Rittenberg, Ann.Phys.(N.Y.) 230250 (1994).

${ }^{15}$ R.B. Stinchcombe, Physica A224 248 (1996).

${ }^{16}$ M. Schulz and S. Trimper, Phys. Rev B 538421 (1996); Phys.Rev E 576398 (1998).

${ }^{17}$ M. Schulz and S. Trimper Int J.Mod.Phys. B 112927 (1997).

${ }^{18} \mathrm{~S}$. Artz and S. Trimper submitted to Phys.Lett A.

${ }^{19}$ M. Schulz and S. Trimper, Phys. Lett. A 216235 (1996).

${ }^{20}$ M. Schulz and S. Trimper, Phys. Lett. A 235113 (1997).

${ }^{21}$ M.Kac in Statistical Physics, Phase Transitions and Superfluidity ed. M.Chétien et al Vol.1 (Gordon and Breach, New York, 1968).

${ }^{22}$ P.C. Hohenberg and B.I. Halperin, Rev.Mod.Phys. 49435 (1977).

23 J.M. Gonzalez-Miranda, P.L. Garrido, J.Marro, and J.L. Lebowitz, Phys. Rev.Lett. 59 1934 (1987).

${ }^{24}$ R.Dickman, Phys.Lett. A 122463 (1987).

25 T.Tomé and M.J. de Oliveira, Phys.Rev A 406643 (1989). 